\title{
DIFFERENCE EQUATIONS AND LETTENMEYER'S THEOREM
}

\author{
W. J. FITZPATRICK, L. J. GRIMM, AND L. M. HALL \\ Department of Mathematics and Statistics, \\ University of Missouri-Rolla, Rolla, MO 65409-0020 \\ Dedicated to Allan Peterson in celebration of his 60th birthday
}

\begin{abstract}
Many classical results for ordinary differential equations have counterparts in the theory of difference equations, although, in general, the technical details for the difference versions are more involved than the corresponding ones for differential equations. This note surveys material related to a difference analoge of Lettenmeyer's theorem. The projection method of W. A. Harris, Jr., Y. Sibuya, and L. Weinberg, developed to treat certain questions in the analytic theory of ordinary differential equations is used to obtain counterparts for linear difference equations as well as extensions to certain nonlinear differential and difference equations.
\end{abstract}

1. INTRODUCTION. In 1969, the seminal paper of W. A. Harris, Jr., Y. Sibuya, and L. Weinberg [10] provided simplified proofs of several classical results in the analytic theory of ordinary differential equations as corollaries of a projection theorem. Analogous results were obtained by W. J. Fitzpatrick and L. J. Grimm [1] for a system of linear difference equations in a setting which had been used by Harris [7] to develop a Frobenius method for constructing a fundamental set of solutions for a system of linear difference equations of the form

$$
(z-1) \Delta_{-1} y(z)=A(z) y(z) .
$$

Here $\Delta_{-1} y(z)=y(z)-y(z-1)$ and $A(z)$ is an $n \times n$ matrix whose elements have convergent factorial series expansions in some right half-plane. In this note, we discuss these and related results, and indicate some directions for further work.

2. FACTORIAL SERIES and LINEAR EQUATIONS. A series, convergent in some half-plane, having the form

$$
f(z)=f_{0}+\sum_{k=0}^{\infty} \frac{f_{k+1} k !}{z(z+1)(z+2) \cdots(z+k)},
$$

Key words and phrases. linear difference equation, factorial series.

Typeset by $\mathcal{A} \mathcal{M} \mathcal{S}-\mathrm{T}_{\mathrm{E}} \mathrm{X}$ 
is called a factorial series. We also define the factorial function

$$
z^{(k)}=z(z-1)(z-2) \cdots(z-k+1) .
$$

Details on the algebra of factorial series can be found in [1] or [13].

Let $X(\delta)$ denote the set of all complex-valued functions which have absolutely convergent factorial series expansions for $\Re(z) \geq \delta>1$. For $h \in X(\delta)$ with

$$
h(z)=h_{0}+\sum_{k=0}^{\infty} \frac{h_{k+1} k !}{z(z+1) \cdots(z+k)}
$$

define

$$
\|h\|=\left|h_{0}\right|+\sum_{k=0}^{\infty} \frac{\left|h_{k+1}\right| k !}{\delta(\delta+1) \cdots(\delta+k)} .
$$

With the operations of addition and scalar multiplication defined on $X(\delta)$ in the natural way, $(X(\delta),\|\cdot\|)$ is a Banach space isomorphic to $l^{1}$. Let $X_{n}(\delta)$ denote the set of all n-vector functions $f=f(z)=\left(f^{1}(z), f^{2}(z), \ldots, f^{n}(z)\right)^{T}$ whose components are elements of $X_{n}(\delta)$. With the norm defined by

$$
\|f\|_{n}=\sum_{i=1}^{n}\left\|f^{i}\right\|,
$$

$\left(X_{n}(\delta),\|\cdot\|\right)$ is also a Banach space.

The following result is a difference version of the Harris-Sibuya-Weinberg theorem.

Theorem 1 [1]. Let $A(z)$ be an $n \times n$ matrix whose elements belong to $X(\delta)$ for some $\delta>1$. Let $D=\operatorname{diag}\left(d_{1}, d_{2}, \ldots, d_{n}\right)$, with each $d_{i}$ equal to either 1 or 2 . Denote by $(z-1)^{(D)}$ the matrix diag $\left((z-1)^{\left(d_{1}\right)},(z-1)^{\left(d_{2}\right)}, \ldots,(z-1)^{\left(d_{n}\right)}\right)$. Then for each positive integer $N$, sufficiently large, and for each vector-valued function $\phi(z)$ such that $(z-1)^{(D)} \phi(z)$ is a polynomial of degree $N$, there exists a vector polynomial $f(z ; \phi)$, which also depends on $A$ and $N$, of degree $N-1$ such that the linear difference system

$$
(z-1)^{(D)} \Delta_{-1} y(z)=A(z) y(z)+f(z ; \phi)
$$

has a factorial series solution $y(z) \in X_{n}(\delta)$. Further, $f$ and $y$ are linear and homogeneous in $\phi$, and $(z-1)^{(D)}(y-\phi)=O\left((z-1)^{-N}\right)$ as $z \rightarrow \infty$ in $\Re(z)>\delta$.

The proof is an application of the Banach fixed point theorem. The polynomials involved are represented in factorial polynomial form, and the statement of the theorem in [1] involves factorial polynomials for this reason. The next three results are corollaries of Theorem 1. 
Corollary 2. Let $d$ denote the number of the $d_{j}$ which equal 2 . Then the system

$$
(z-1)^{(D)} \Delta_{-1} y(z)=A(z) y(z)
$$

has at least d linearly independent factorial series solutions in $X_{n}(\delta)$.

Remarks.

1. Corollary 2 is a difference equation analogue of a theorem of $F$. Lettenmeyer (see [4]).

2. If $d_{j}=2$ for $j=1,2, \ldots, n$, then (2) has $n$ linearly independent convergent factorial series solution. this case is analogous to a linear differential system with an ordinary point at $z=\infty$.

3. By the use of Waring's formula [13], the case where some $d_{j}>2$ can be reduced to $d_{j}=2$ before using Theorem 1, making Theorem 1 applicable to all cases where each $d_{j}$ is a positive integer.

4. A result of Harris and H. L. Turrittin [11] on factorial series reciprocals of factorial series permits reduction of a system of the form

$$
z^{k} F(z) \Delta_{-1} y(z)=A(z) y(z),
$$

where $F$ and $A$ have factorial series representations and $k$ is a positive integer, to a system of the form considered here.

5. Corollary 2 can also be obtained by a degree-theoretic argument [6].

In case a formal factorial series solution of (1) exists, we have the following difference version of the theorem of Sauvage (note that equation (1) is a difference analogue of a differential equation with a regular singular point at infinity).

Corollary 3. Let

$$
y(z)=y_{0}+\sum_{k=0}^{\infty} \frac{y_{k+1} k !}{z(z+1) \cdots(z+k)}
$$

be a formal solution of (1) in the sense of equality of formal factorial series. Then $y \in X_{n}(\delta)$ and thus every formal factorial series solution is convergent.

Corollary 4. Let $A(z)$ be an $n \times n$ matrix, with elements in $X(\delta)$, having the factorial series expansion

$$
A(z)=A_{0}+\sum_{k=0}^{\infty} \frac{A_{k+1} k !}{z(z+1) \cdots(z+k)} .
$$

Let $n_{\lambda}$ be the number of linearly independent eigenvalues of $A_{0}$ corresponding to the eigenvalue $\lambda$. Then the number $N_{\lambda}$ of linearly independent solutions of the system (1) which have the form

$$
y(z)=\frac{\Gamma(z)}{\Gamma(z-\lambda)} w(z),
$$

where $w(z) \in X_{n}(\beta), \beta=\max (\delta, 1+\Re(\lambda))$, satisfies

$$
\max \left(n_{\lambda}, n_{\lambda-1}, \ldots\right)<N_{\lambda}<n_{\lambda}+n_{\lambda-1}+\cdots .
$$

The general result corresponding to the Frobenius series representation for solutions at regular singular points is also obtained using a Harris-Sibuya-Weinberg type procedure. 
Theorem 5. Let $A(z)$ be an $n \times n$ matrix as in Corollary 4. If the distinct eigenvalues of $A_{0}, \lambda_{1}, \ldots, \lambda_{k}, k \leq n$, do not differ by integers, then (1) has $n$ linearly independent solutions of the form

$$
\frac{\Gamma(z)}{\Gamma\left(z-\lambda_{i}\right)}\left[y_{i}^{[1]}(z)+y_{i}^{[2]}(z) \psi_{1}(z)+y_{i}^{[3]}(z) \psi_{2}(z)+\cdots+y_{i}^{\left[s_{i}\right]}(z) \psi_{s_{i}-1}(z)\right]
$$

where $\psi_{1}(z)=\frac{-\Gamma(z)}{\Gamma(z+1)}$ is the digamma function, $\psi_{j}(z)=(-1)^{j} \Gamma(z) \frac{d}{d z}\left(\frac{1}{\Gamma(z)}\right), j=$ $2, \ldots, s_{i}-1$, and $s_{i}$ is less than or equal to the algebraic multiplicity of the eigenvalue $\lambda_{i}$. Each $y_{i}^{[j]}(z)$ is in $X_{n}(\beta)$, where $\beta=\max \left\{\delta, 1+\Re\left(\lambda_{i}\right)\right\}$.

Remarks.

1. The case where $A_{0}$ has eigenvalues differing by integers may be reduced to the above case using a theorem of Harris [7].

2. The proof of Theorem 5 given in [1] follows an argument for differential equations provided by W. Walter [12]. Harris [9] also obtained that result by a different argument.

\section{NONLINEAR EQUATIONS.}

Consider the nonlinear system

$$
(z-1)^{(D)} \Delta_{-1} y(z)=f(z, y(z)),
$$

where

(i) $y=\left(y^{1}, y^{2}, \ldots, y^{n}\right)^{T}$ and $f=\left(f^{1}, f^{2}, \ldots, f^{n}\right)^{T}$ are n-vectors and $f$ has the expansion

$$
f(z, y(z))=f_{0}(z)+A(z) y(z)+\sum_{|p| \geq 2} f_{p}(z) y^{p}
$$

with $A$ an $n \times n$ matrix, $p$ a set of nonnegative integers $p_{1}, \ldots, p_{n}$, and

$$
y^{p}=\prod_{i=1}^{n}\left(y^{i}(z)\right)^{p_{i}}, \quad|p|=\sum_{i=1}^{n}\left|p_{i}\right| .
$$

(ii) The vectors $f_{0}$ and $f_{p}$ have absolutely convergent factorial series representations in the half-plane $\Re(z) \geq \delta>1$.

(iii) There exists a constant $\eta>0$ such that the series $\sum_{|p| \geq 2} f_{p}(z) u^{p}$ converges uniformly in $z$ and $u$ for $\Re(z) \geq \delta$ for all vectors $u=\left(u^{1}, \ldots, u^{n}\right)^{T}$ satisfying $\|u\|<\eta$.

A nonlinear version [2] of Theorem 1 can be used to provide results for equation (3) corresponding to the corollaries of Theorem 1. For example, the analogue of the Lettenmeyer theorem is as follows. 
Theorem 6. Let $D$ be as in Theorem 1 with $d=\operatorname{trace}(D)$ and let $f$ satisfy (4) with $F_{0}(z) \equiv 0$. Then the system (3) has at least a $(d-n)$-parameter algebroid family of factorial series solutions in $X_{n}(\delta)$ for some $\delta$ sufficiently large.

A corresponding result for differential equations is given in [8].

\section{NOTES and QUESTIONS.}

(a) The proof of Corollary 2 does not extend to any case where any $d_{i}=0$, which would correspond to an irregular singular point at infinity of a corresponding differential equation. In the differential equations case, however, Lettenmeyer's theorem can apply, and either the Harris-Sibuya-Weinberg method or degree theory [12] will provide a proof. Corollary 2 also can be obtained by a degree-theoretic method [6], but again, the proof does not extend to any irregular singular case. (Actually, the "restrictive hypothesis" mentioned in a remark at the end of $[6]$ is an understatement - the hypothesis essentially removes the singularity.) Thus, the question remains: Is there a Lettenmeyer theorem for any nontrivially irregular singular system of difference equations?

(b) In the differential equations case, Grimm and Hall [5] used a degree-theoretic approach to develop an alternative theorem to solve a problem posed by Turrittin [14], which was based on an example of Briot and Bouquet dating back as far back as 1845 . In the factorial series setting, an analogous alternative theorem would answer the question:

Given a difference equation

$$
(z-1)^{(D)} \Delta_{-1} y(z)=A(z) y(z)+g(z),
$$

where $g(z) \in X_{n}(\delta)$, under what conditions will there exist a solution convergent in some right half-plane?

In the special case where all the $d_{i}$ are equal to 1 and $A_{0}$ has no nonpositive integer eigenvalue, a result of Grimm and Fitzpatrick [2] guarantees a solution in $X_{n}(\delta)$ for $\delta$ sufficiently large under no additional hypotheses on g. A differential equations analogue of this result is in the paper of $\mathrm{K}$. O. Friedrichs [3].

(c) The recent development of the theory of dynamic equations on time scales by S. Hilger (who started it all), A. C. Peterson, M. Bohner, and others has already done much to connect the theories of differential equations and difference equations. Perhaps some version of the results in this paper can be developed on time scales.

\section{REFERENCES}

1. W. J. Fitzpatrick and L. J. Grimm, Convergent factorial series solutions of linear difference equations, J. Differential Equations 29 (1978), 345-361.

2. W. J. Fitzpatrick and L. J. Grimm, On solutions of nonlinear difference systems, Funkcial. Ekvac. 23 (1980), 17-23.

3. K.O. Friedrichs, Advanced Ordinary Differential Equations, Gordon and Breach, New York, 1965. 
4. L. J. Grimm and L. M. Hall, Holomorphic solutions of functional differential systems near singular points, Proc. Amer. Math. Soc. 42 (1974), 167-170.

5. L. J. Grimm and L. M. Hall, An alternative theorem for singular differential systems, J. Differential Equations 18 (1975), 411-422.

6. L. J. Grimm and W. D. Noble, Factorial series solutions of linear difference equations, Differential Equations and Applications, Rousse (1987), 695-699.

7. W. A. Harris, Jr., Linear systems of difference equations, Contrib. Differential Equations 1 (1963), 489-518.

8. W. A. Harris, Jr., Holomorphic solutions of nonlinear differential equations at singular points, SIAM Stud. Appl. Math. 5 (1969), 184-187.

9. W. A. Harris, Jr., Logarithmic solutions of linear differential systems with a singularity of the first kind, Applicable Analysis 8 (1978), 171-174.

10. W. A. Harris, Jr., Y. Sibuya and L. Weinberg, Holomorphic solutions of linear differential systems at singular points, Arch. Rational Mech. Anal. 35 (1969), 245-248.

11. W. A. Harris, Jr. and H. L. Turrittin, Reciprocals of inverse factorial series, Funkcial. Ekvac. 6 (1964), 37-46.

12. Ju. F. Korobeinik, Normal solvability of linear differential equations in the complex plane, Math. USSR Izvestija 6 (1972), 445-466.

13. L. M. Milne-Thompson, The Calculus of Finite Differences, MacMillan and Co., London, 1933.

14. H. L. Turrittin, My mathematical expectations, Symposium on Ordinary Differential Equations, Lecture Notes in Mathematics, vol. 312, Springer-Verlag, 1973, pp. 1-22.

15. W. Walter, Gewöhnliche Differentialgleichungen, Springer-Verlag, Berlin, 1972. 